\title{
An Elementary Approach to the Monster
}

\section{Christopher S. Simons}

1. INTRODUCTION. Finding the finite simple Fischer-Griess Monster group $\mathbb{M}$ of order

$$
808017424794512875886459904961710757005754368000000000
$$

is one of the most spectacular and mysterious mathematical achievements of the past fifty years. Unfortunately the various standard approaches to the Monster are notoriously difficult to learn. This paper presents a relatively elementary approach to the Monster. We describe a construction of the smallest non-Abelian finite simple group (of order 60), and show that it very closely parallels a construction of $\mathbb{M} \times \mathbb{M}$.

In section 2, before we begin our main discussion, we give a quick overview of the Monster both as a finite simple group and with respect to its most startling properties. This is done to allow those readers who have only a passing acquaintance with the Monster to get a better sense of its role in mathematics. Despite its brevity, this section has a vast scope, so we refer the reader to other sources for more comprehensive treatments (see [1], [2], or [6]). In section 3 we start with the tetrahedral graph and use Coxeter groups, along with certain additional relations, to obtain a group presentation for the finite simple group $A_{5}$. Then in section 4 we see that this method, when applied to different initial graphs, leads not only to the square $\mathbb{M} \times \mathbb{M}$ of the elusive Monster group, but to other finite simple groups as well.

2. A QUICK OVERVIEW OF THE MONSTER. Hints of the Monster's existence first arose during the intense search for new finite simple groups that took place during the 1960s and 1970s and occurred simultaneously with the effort to classify finite simple groups. In fact, it was only after the classification was essentially complete that Robert Griess proved the existence of the Monster. According to the classification, every finite simple group falls into one of the following five families:

(1) the cyclic groups $C_{p}$ for $p$ prime;

(2) the alternating groups $A_{n}$ for $n \geq 5$ ( $A_{n}$ is the group of all even permutations on $n$ letters; $A_{5}$, which has order 60 , is the smallest such group);

(3) the matrix groups $L_{n}(q), O_{n}^{ \pm}(q), U_{n}(q)$, and $S p_{n}(q)$ (these are simple groups derived from the $n$-dimensional linear, orthogonal, unitary, and symplectic matrix groups over the field of order $q$ );

(4) the exceptional groups of Lie-type;

(5) twenty-six "sporadic" groups (including the Monster).

The existence of the first four infinite families is well understood using number theory, basic permutation group theory, linear algebra, and Lie geometry. The fifth family contains the twenty-six finite simple groups that simply do not belong to any other family. The Monster is the largest of these sporadic groups, and nineteen other sporadic groups appear as homomorphic images of subgroups of the Monster. Despite a great deal of work, the sporadic groups remain mysterious, so a better understanding of the Monster would improve our understanding of finite groups in general. Announced 
in the early 1980s, the proof of the classification of finite simple groups stretches the traditional notions of proof. The proof involves some very difficult and specialized mathematics spanning many thousands of pages, not all of which have been published. To correct this deficiency, Richard Lyons and Ronald Solomon are leading an ambitious and active program to produce a revised and complete proof of classification. As of this writing, Michael Aschbacher and Stephen Smith are still in the process of revising a 1196-page preprint on the so-called quasi-thin case in order to close a gap in the original proof. Although there is little doubt that the classification is correct, there remains great interest in obtaining a new and more efficient proof.

However, it is not the Monster's role in group theory that has drawn the most attention to it. Of even greater interest is the Monster's miraculous "moonshine" phenomenon. In the late 1970s, John McKay made a surprising connection between the character table of the Monster and modular functions. The character table of the Monster is a $194 \times 194$ array of complex numbers that are the traces of its irreducible representations on the conjugacy classes of the Monster. The first three entries of the first column (corresponding to the identity conjugacy class) of the table are 1, 196883, and 21296876. McKay asked whether the equality $1+196883=196884$ makes a real connection between the character table of the Monster and the elliptic modular function

$$
j(z)=\frac{1}{q}+196884 q+21493760 q^{2}+\cdots
$$

(where $q=e^{2 \pi i z}$ ). Although at first blush unbelievable, John H. Conway, Simon Norton, and John Thompson showed that this connection persists. The term "moonshine" reflects both the mysterious, or dimly lit, nature of this connection, as well as its somewhat illicit origin in numerology. Not only by dint of the relationship between the first column of the character table and the elliptic modular function (note that

$$
1+196883+21296876=21493760
$$

and similarly for the higher order coefficients of $j$ ), but also through consideration of the other columns, a surprisingly strong linkage comes to light between the conjugacy classes of the Monster and a remarkable class of modular functions. As such, moonshine connects two mathematical areas that seemingly should not be related. In 1998, Richard Borcherds received the Fields Medal for his work on the proof of these moonshine conjectures. Moreover, he established the connection between the Monster and modular functions by exploiting information about vertex operator algebras from conformal field theory. Instead of simpliflying the matter, Borcherds succeeded in heightening interest in Monster by exposing hidden ties between three disparate areas of mathematics: finite simple groups, modular functions, and vertex operator algebras.

Both for its role in finite group theory and from its association with the moonshine conjectures the Monster now occupies a prominent position in mathematics. The presentation in the remainder of this paper provides an elementary way of accessing this very nonelementary object. It is hoped that it, or other simple approaches, may eventually contribute to more satisfying explanations for the subjects that we have just discussed.

\section{FROM THE TETRAHEDRAL GRAPH TO $A_{5}$.}

The tetrahedral Coxeter group. We start our search for non-Abelian finite simple groups by considering the tetrahedral graph $K_{4}$. This is the complete graph with four nodes that we refer to as the 4-node diagram (Figure 1). 


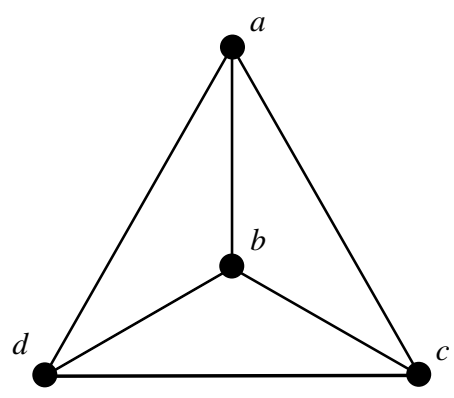

Figure 1. The 4-node diagram $K_{4}$.

We now want to attach a group to the 4-node diagram. If we consider the symmetry group of this graph, we find that it is isomorphic to the symmetric group $S_{4}$ of order $4 !=24$, which is too small to contain any non-Abelian finite simple groups. To obtain something, we must therefore use another method to attach a group to the 4-node diagram. We do this by considering its Coxeter group. The theory of Coxeter groups dates from the early 1930s (an excellent reference on Coxeter theory is [4]). The Coxeter group of a graph is the group generated by (nontrivial) elements $r_{i}$ corresponding to the nodes of the graph subject only to the following relations:

(i) $r_{i}^{2}=1$ for each node $i$;

(ii) $\left(r_{i} r_{j}\right)^{2}=1$ whenever nodes $i$ and $j$ are not joined by an edge;

(iii) $\left(r_{i} r_{j}\right)^{3}=1$ whenever nodes $i$ and $j$ are joined by an edge.

We can think of the Coxeter group of an $n$-node graph as being the largest group generated by $n$ distinct involutions (elements of order 2) subject to the conditions that the product of two involutions corresponding to unjoined nodes has order 2 and the product of two involutions corresponding to joined nodes has order 3.

Any group $G$, such as a Coxeter group, whose relations are all of even length (where the length of a relation is the number of generators, counting multiplicities, that it contains) has a normal subgroup of index two consisting of those elements that can be written as a product of evenly many generators. We call this the even subgroup of $G$ and denote it by $\frac{1}{2} G$.

Now let $G$ be the the Coxeter group corresponding to the 4-node diagram, a group known for obvious reasons as the tetrahedral Coxeter group. We note that $G$ is then generated by four involutions, where the product of any two distinct generators has order 3. Unfortunately, $G$ is an infinite group. In fact, it even has elements of infinite order. We proceed by finding some of these elements. To do so, we first consider certain infinite subgroups of $G$.

For a graph $\Gamma$, we define a free $n$-gon in $\Gamma$ to be a cycle of length $n$ such that each node in the cycle appears only once and is adjacent to exactly two other nodes in the cycle. A maximal free $n$-gon in $\Gamma$ is a free $n$-gon such that $\Gamma$ contains no free $m$-gon with $m>n$.

For the 4-node diagram, the maximal free $n$-gons are 3 -gons corresponding to the triangular faces of the tetrahedron. We note that these 3-gons are equivalent to each other under the symmetry group of the 4-node diagram. We now take any of these 3-gons, say the face with nodes $a, b$, and $c$ (Figure 2) and consider the Coxeter group $H$ generated by the involutions $r_{a}, r_{b}$, and $r_{c}$ corresponding to the nodes of this maximal 3 -gon. Fortunately it is not difficult to understand the structure of $H$. Indeed, Coxeter theory tells us that all Coxeter groups are isomorphic to groups generated by geometric 


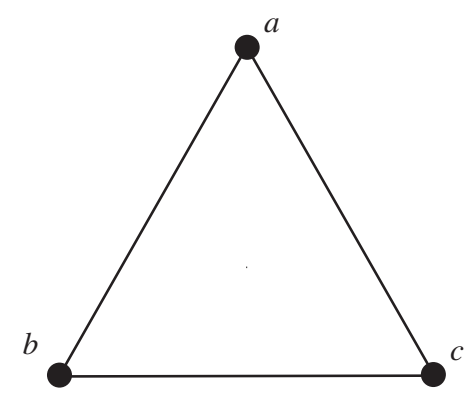

Figure 2. A maximal free 3-gon in $K_{4}$.

reflections, so it suffices to see how $H$ acts as a reflection group. It does so quite nicely on a two-dimensional plane.

At this point it becomes notationally convenient to identify the elements $r_{a}, r_{b}$, and $r_{c}$ by the abbreviated names $a, b$, and $c$. These elements then correspond to the reflections in the lines (in a two-dimensional plane) indicated in Figure 3. (Be aware that we are using the same letter to denote a node, a generator of the Coxeter group, a line in a two-dimensional plane, and the reflection of the plane in that line.) We note that these reflections satisfy the required Coxeter relations. Reflections naturally have order 2, and since the angle between any two of the lines for $a, b$, and $c$ is 60 degrees, the product of any two of the reflections is a rotation by 120 degrees about the intersection of the associated lines, and therefore has order 3.

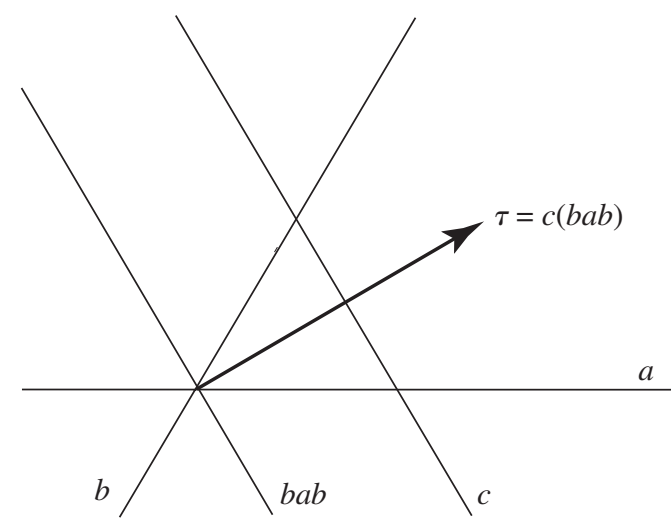

Figure 3. The reflection group $H$.

We now find a new element of $H$ by conjugating the reflection $a$ by the reflection $b$. This is the element $b a b^{-1}$, which can be rewritten as $b a b$ since $b=b^{-1}$. If we take the image of the line $a$ when reflected in the line $b$ we obtain a new line denoted $b a b$ in Figure 3. The group element $b a b$ then acts as the reflection in this line. We now let $\tau$ be the element obtained by first performing the reflection $b a b$ and then the reflection $c$. So $\tau=c(b a b)=c b a b$. Examining the action of $\tau$ on the plane, we find that it translates all points of the plane in the direction indicated in Figure 3. The reader is encouraged to check the foregoing claims by following individual points under the indicated reflections.

Translations in the plane have infinite order, so $o(\tau)=\infty$. Since we wish to deal with finite groups, we must do something to $\tau$. We note that there are infinitely many 
translations in $H$ and that they form a lattice isomorphic to $\mathbb{Z}^{2}$. A translation in $H$ is said to be primitive (relative to $H$ ) if it cannot be obtained by raising another translation from $H$ to a power greater than one.

A very finite group. The easiest way to deal with the infinite order element $\tau$ in order to arrive at a finite group is to insist that $\tau$ have order 1 ! While this might sound at first to be totally outrageous, what we really have in mind is introducing into $H$ a new relation, namely, the relation $\tau=1$. We call this deflating the translation. Since all primitive translations in $H$ are conjugate, this has the effect of deflating all translations in $H$, so our particular choice of $\tau$ turns out to be unimportant.

As an aside we note that the relation $\tau=1$ is equivalent to $c=b a b$. Therefore imposing this relation on the group $H=\langle a, b, c\rangle$ generated by $a, b$, and $c$ makes it isomorphic to the finite Coxeter group $\langle a, b\rangle$ generated by $a$ and $b$. The group $\langle a, b\rangle$ consists of the reflections $a, b$, and $b a b$ along with the rotations through 0,120 , and 240 degrees about the point of intersection of the lines determining $a$ and $b$. As such it is isomorphic to the dihedral group of order 6 , or equivalently, to $S_{3}$. Something very different will occur when we move to a broader context.

We can define a new group $G_{1}$ to be the group generated by the generators of the tetrahedral Coxeter group $G$, subject to both the Coxeter relations and the deflation relations stating that the translations $\tau$ arising from all four of the maximal free 3gons in $K_{4}$ have order 1 . These deflations are a bit too powerful to produce anything interesting. The group $G_{1}$ has only two elements, and its even subgroup $\frac{1}{2} G_{1}$ is the trivial group of order 1 . This can be seen by noting that the deflations make $c=b a b$ and $d=b a b$, whence $c=d$. Similarly all four generating involutions of $G_{1}$ are equal.

The smallest non-Abelian simple group. Of course in the deflation process we did not have to insist that $\tau$ have order 1 . We could just as well require it to be of some specified finite order. For instance, we can try imposing the relation $\tau^{2}=1$. This is called biflating the translation. Again, because all primitive translations in $H$ are all conjugate, this has the effect of biflating all translations in $H$.

We now define $G_{2}$ to be the group generated by the generators of $G$, subject to both the Coxeter relations and the biflation relations demanding that all translations $\tau$ arising from the maximal free 3-gons in $K_{4}$ have order 2 . This time we find that $G_{2}$ has order 120 and is isomorphic to $S_{5}$, the symmetric group on five letters. The even subgroup $\frac{1}{2} G_{2}$ is isomorphic to $A_{5}$, the alternating group on five letters. Of course, $A_{5}$ is the smallest non-Abelian simple group.

We can understand the structure of $G_{2}$ by noting that, if we map $a$ to the permutation (15), $b$ to (25), $c$ to (35), and $d$ to (45), then these images of $a, b, c$, and $d$ generate $S_{5}$ and all the required relations of $G_{2}$ are satisfied by them. For example,

$$
\tau=c b a b=(35)(25)(15)(25)=(12)(35)
$$

does indeed have order 2. It follows that $S_{5}$ is a homomorphic image of $G_{2}$. To show that $G_{2}$ is isomorphic to $S_{5}$, we need to check only that $G_{2}$ is not bigger than $S_{5}$. In other words we want $\left|G_{2}\right| \leq 120\left(=5 !=\left|S_{5}\right|\right)$. This is done in the next subsection using a coset enumeration argument. At this point the reader unconcerned with technical details of this kind can skip ahead to section 4.

Coset enumeration. We start by recalling that the subgroup $\langle a, b\rangle$ of $G_{2}$ has order at most 6 . We now consider the subgroup $\langle a, b, c\rangle$. The relation $\tau^{2}=(c b a b)^{2}=1$ 
implies that

$$
c b a b=(c b a b)^{-1}=b^{-1} a^{-1} b^{-1} c^{-1}=b a b c .
$$

Multiplying this relation on the left by $b$ gives the identity $c b a=b a b c b$. In a similar manner we obtain $c a b=$ abaca. Using these identities, in tandem with the Coxeter relations, we find that any word in $a, b$, and $c$ can be rewritten so that if $c$ occurs in the word at all, $c$ appears only in one of the two rightmost positions in the word. As a result, $\langle a, b, c\rangle$ has at most four right cosets corresponding to the subgroup $\langle a, b\rangle$ : $\langle a, b\rangle 1,\langle a, b\rangle c,\langle a, b\rangle c a$, and $\langle a, b\rangle c b$. We infer that $|\langle a, b, c\rangle| \leq 4|\langle a, b\rangle| \leq 24$.

We now consider $G_{2}=\langle a, b, c, d\rangle$. Using a similar pattern of relations (starting from the other 3-gons of $K_{4}$ ), we discover that any word in $a, b, c$, and $d$ can be rewritten as a word with $d$ appearing, if at all, only in one of the two rightmost positions. Consequently $\langle a, b, c, d\rangle$ splits into at most five right cosets of $\langle a, b, c\rangle:\langle a, b, c\rangle 1$, $\langle a, b, c\rangle d,\langle a, b, c\rangle d a,\langle a, b, c\rangle d b$, and $\langle a, b, c\rangle d c$. Therefore $\left|G_{2}\right| \leq 5|\langle a, b, c\rangle| \leq$ 120 as desired.

The family of alternating simple groups. In fact, if $n \geq 4$ and we start with $K_{n}$, the complete graph with $n$ nodes $a_{1}, a_{2}, \ldots, a_{n}$, then the corresponding biflation group $G_{2}^{(n)}$ is isomorphic to $S_{n+1}$ and the even subgroup $\frac{1}{2} G_{2}^{(n)}$ is isomorphic to the simple alternating group $A_{n+1}$. This can be proved by extending the previous arguments. By assigning the permutations $(1, n+1),(2, n+1), \ldots,(n, n+1)$ to the nodes of $K_{n}$, we can easily see that $S_{n+1}$ is a homomorphic image of $G_{2}^{(n)}$. We then need to show only that $\left|G_{2}^{(n)}\right| \leq(n+1) !\left(=\left|S_{n+1}\right|\right)$. This is done by induction. We have already verified the base case $n=4$. Assuming that $\left|G_{2}^{(n)}\right| \leq(n+1)$ !, we extend $K_{n}$ to $K_{n+1}$ by adjoining a new node $a_{n+1}$, and by an argument similar to earlier ones we establish that any word in $a_{1}, a_{2}, \ldots, a_{n+1}$ containing $a_{n+1}$ can be rewritten to make $a_{n+1}$ appear only in one of the two rightmost positions, meaning that $G_{2}^{(n+1)}$ breaks into at most $n+2$ right cosets relative to $G_{2}^{(n)}$. Therefore

$$
\left|G_{2}^{n+1}\right| \leq(n+2)\left|G_{2}^{(n)}\right| \leq(n+2)(n+1) !=(n+2) !,
$$

as desired.

\section{THE MONSTER.}

The 26-node diagram. Instead of starting with the tetrahedral 4-node diagram we now start with a graph that we call the 26-node diagram and denote it by $I_{3}$ for reasons that we explain shortly. The 4-node diagram $K_{4}$ is a highly symmetric graph with four nodes and valence (i.e., the number of edges incident at each node) three. The 26-node diagram $I_{3}$ is a highly symmetric graph with twenty-six nodes and valence four. The exact details of the 26-node diagram are somewhat complicated and will not be needed in the sequel. In the interest of completeness we provide them for the interested reader.

The 26-node diagram is the incidence graph $\operatorname{Inc}\left(\mathbb{P}_{3}\right)$ of the projective plane $\mathbb{P}_{3}$ of order three. The projective plane $\mathbb{P}_{3}$ can be thought of as the finite geometry consisting of thirteen points and thirteen lines with four points on each line and four lines passing through each point. Each pair of points determines a unique line and any two distinct lines intersect at a unique point. The incidence graph of $\mathbb{P}_{3}$ has twenty-six nodes (one for each of the thirteen points and one for each of the thirteen lines). Two nodes are joined precisely when one corresponds to a line, the other corresponds to a point, and the point lies on the line. 


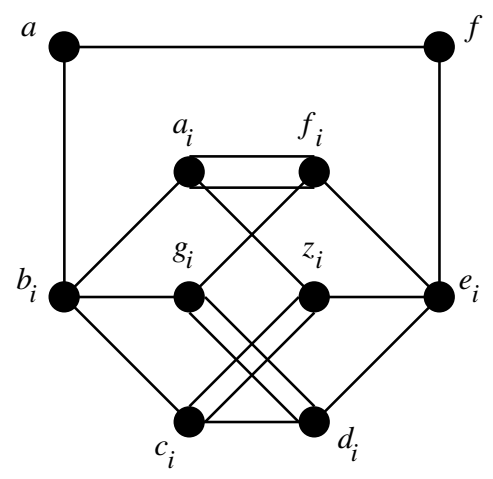

Figure 4. A representation of the 26-node diagram.

The 26-node diagram is represented in Figure 4. The indices $i$ range over $\{1,2,3\}$, so some of the nodes that appear in the figure correspond to three different nodes of the 26-node diagram. Nodes $a$ and $f$ are joined to each other as well as to $b_{1}, b_{2}$, and $b_{3}$ and $e_{1}, e_{2}$, and $e_{3}$, respectively. Otherwise single edges between nodes indicate that the corresponding nodes of the 26-node diagram are joined just if they have the same indices. For example, $b_{1}$ is joined to $g_{1}, b_{2}$ is joined to $g_{2}$, and $b_{3}$ is joined to $g_{3}$. Double edges between nodes indicate that the corresponding nodes of the 26-node diagram are joined just when their indices differ. For example, $a_{1}$ is joined to $f_{2}$ and $f_{3}, a_{2}$ is joined to $f_{1}$ and $f_{3}$, and $a_{3}$ is joined to $f_{1}$ and $f_{2}$.

The bimonster. We now apply the same process used on the 4-node diagram to the 26-node diagram. Further details can be found in [3]. Let $G$ be the Coxeter group associated with $I_{3}$. It is generated by twenty-six involutions and subject to the appropriate Coxeter relations. Again $G$ is an infinite group.

The maximal free $n$-gons in $I_{3}$ are 12 -gons. These free 12 -gons are, in fact, all equivalent under the symmetry group of the 26-node diagram. With each such 12-gon we can associate elements that act as translations on eleven-dimensional Euclidean vector spaces. Let $\tau$ be a (primitive) translation of this type. Then $\tau$ then has infinite order, so we deflate it by imposing the relation $\tau=1$.

We then define $G_{1}$ to be the group generated by the generators of $G$, subject to both the Coxeter relations and the deflation relations requiring that all translations associated with the maximal free 12 -gons in $I_{3}$ have order 1 . It turns out that $G_{1}$ is an enormous finite group with order approximately $10^{108}$. This group is isomorphic to the bimonster group $\mathbb{M}<2$ (the wreathed square of the Monster). The even subgroup $\frac{1}{2} G_{1}$ is isomorphic to the direct product of the Monster with itself (i.e., $\mathbb{M} \times \mathbb{M}$ ). The elements of the bimonster $\mathbb{M}<2$ are either elements $(x, y)$ of $\mathbb{M} \times \mathbb{M}$ or have the form $(x, y) \sigma$, where $\sigma$ is a (wreathing) involution satisfying the family of relations $(x, y) \sigma=\sigma(y, x)$. This identification is considerably more difficult to establish than the analogous result for the 4-node diagram. A proof can be found in [3]. It appeals to some deep results from the "monstrous" literature, including the Ivanov-Norton theorem. The connection between the Coxeter group of the 26-node diagram and the bimonster was first made by Simon Norton. The identification of $\frac{1}{2} G_{1}$ with $\mathbb{M} \times \mathbb{M}$ gives us an elementary way of viewing (the square of) the celebrated Monster group.

Beyond. It is of course possible to apply this process to other nice graphs (where a "nice" graph is one that naturally leads to an interesting finite group). The case involving the 14-node incidence graph $I_{2}=\operatorname{Inc}\left(\mathbb{P}_{2}\right)$ of the projective plane of $\mathbb{P}_{2}$ of 
order two is particularly instructive [5]. We provide a brief list outlining some such constructions in Table 1. The groups are listed using the Atlas notation for simple groups [2].

Table 1. From graphs to simple groups.

\begin{tabular}{lcccc}
\hline \# of nodes & graph & $n$-gons & action & even subgroup \\
\hline$n(\geq 4)$ & $K_{n}$ & 3-gons & biflation & $A_{n+1}$ \\
8 & cube & 6-gons & deflation & $O_{5}(3) \cong O_{6}^{-}(2)$ \\
10 & Petersen & 6-gon & deflation & $O_{6}^{-}(2) \cong O_{5}(3)$ \\
14 & $\operatorname{Inc}\left(\mathbb{P}_{2}\right)$ & 8-gons & deflation & $O_{8}^{-}(2)$ \\
26 & $\operatorname{Inc}\left(\mathbb{P}_{3}\right)$ & 12-gons & deflation & $\mathbb{M} \times \mathbb{M}$ \\
\hline
\end{tabular}

\section{REFERENCES}

1. R. E. Borcherds, What is the Monster?, Notices Amer. Math. Soc. 49 (2002) 1076-1077.

2. J. H. Conway, R. T. Curtis, S. P. Norton, R. A. Parker, and R. A. Wilson, Atlas of Finite Groups, Oxford University Press, Eynsham, U.K., 1985.

3. J. H. Conway and C. S. Simons, 26 implies the bimonster, J. Algebra 235 (2001) 805-814.

4. H. S. M. Coxeter and W. O. J. Moser, Generators and Relations for Discrete Groups, 4th ed., SpringerVerlag, Berlin, 1980.

5. C. S. Simons, Deflating infinite Coxeter groups to finite groups, in Proceedings on Moonshine and Related Topics, J. McKay and A. Sebbar, eds., CRM Proc. Lecture Notes, no. 30, American Mathematical Society, Providence, 2001, pp. 223-229.

6. R. Solomon, The shape of the classification of the finite simple groups, in Groups and Combinatorics-in Memory of Michio Suzuki, E. Bannai, H. Suzuki, H. Yamaki and T. Yoshida, eds., Adv. Stud. Pure Math., no. 32, Mathematical Society of Japan, Tokyo, 2001, pp. 379-390.

CHRISTOPHER S. SIMONS did his undergraduate work at McGill University and his graduate work at Princeton University, where his advisor was John H. Conway. With the support of an NSERC fellowship, he held postdoctoral positions at McGill University, Concordia University, and the University of California, San Diego. He shared earlier versions of this paper with his modern algebra students at Rowan University, where he is assistant professor of mathematics. His research has centered on finite groups, including the Monster. Recently he has become interested in the chirality of integer triples. His other interests include spending time with his wife Laura and daughters Caty and Chelsea.

Department of Mathematics, Rowan University, Glassboro, NJ 08028

simons@rowan.edu 